\title{
Analytical wave functions for atomic quantum-defect theory
}

\author{
V. Alan Kostelecký* and Michael Martin Nieto ${ }^{\dagger}$ \\ Theoretical Division, Los Alamos National Laboratory, University of California, Los Alamos, New Mexico 87545
}

(Received 12 August 1985)

\begin{abstract}
We present an exactly solvable effective potential that reproduces atomic spectra in the limit of exact quantum-defect theory, i.e., the limit in which, for a fixed $l$, the principal quantum number is modified by a constant: $n^{*}=n-\delta(l)$. Transition probabilities for alkali atoms are calculated using the analytical wave functions obtained and agree well with accepted values. This allows us to make phenomenological predictions for certain unknown transition probabilities. Our analytical wave functions might serve as useful trial wave functions for detailed calculations.
\end{abstract}

Rydberg noted ${ }^{1}$ that the spectra of many atoms have series given approximately by

$$
E_{n} *=-E_{0} / n^{* 2}
$$

In modern notation, $E_{0}$ is a constant with dimensions of energy and $n^{*}$ is

$$
n^{*}=n-\delta(l),
$$

where $n$ is a positive integer, the principle quantum number, and $\delta(l)$ is approximately constant for a given fixed orbital quantum number $l, l \geqq 0$. Spectra given by Eqs. (1) and (2) are called Rydberg series, and their study is known as quantum-defect theory.

The quantum defect $\delta(l)$ phenomenologically models the effective charge, generated by the $Z-1$ core electrons and the nucleus of charge $Z$, by shifting the energy eigenvalues away from the hydrogenic values. For example, the low-lying $s$ orbitals of lithium may be described ${ }^{2}$ by the values $n^{*}=1.588,2.596,3.598$, etc., rather than by the hydrogenic values $n=2,3,4$, etc. In the modern approach to quantum-defect theory, ${ }^{3}$ the quantum defect $\delta(l)$ is defined as the phase shift of an exact atomic wave function relative to Coulomb wave functions beyond a core radius.

As Cowan has summarized, ${ }^{3}$ the standard approach to quantum-defect theory usually proceeds either with de-

$$
L_{n}^{(\alpha)}(x)=\sum_{p=0}^{n}(-x)^{p} \Gamma(n+\alpha+1) /[p ! \Gamma(p+\alpha+1) \Gamma(n-p+1)],
$$

instead of the related $L_{n+l}^{2 l+1}(y / n)$ often used in quantum mechanics, ${ }^{4}$ which are defined by

$$
L_{n}^{j}(x)=d^{j} L_{n}(x) / d x^{j},
$$

where $L_{n}$ are the ordinary Laguerre polynomials. Equation (7) is valid for integer $j$ only. This distinction will be important in the following discussion.

Returning to exact quantum-defect theory, we propose the following equation ${ }^{5}$ to model the effective radial equation of the valence electron in the presence of the quantum defect:

$$
\left[-d^{2} / d y^{2}-1 / y+l(l+1) / y^{2}+V_{\text {eff }}(y)+1 / 4 n^{* 2}\right] y R_{n} *_{l}^{*}(y)=0 .
$$

Here, $V_{\text {eff }}(y)$ is chosen to satisfy

$$
\begin{aligned}
& l(l+1) / y^{2}+V_{\mathrm{eff}}(y)=l^{*}\left(l^{*}+1\right) / y^{2}, \\
& l^{*}=l-\delta(l)+I(l),
\end{aligned}
$$


for integer $I(l)$. The range of possible integers $I(l)$ will be discussed later. A glance at the hydrogen equation shows that the potential $V_{\text {eff }}(y)$ yields a solution with the same functional form as Eq. (4) but with noninteger values of $n *$ and $l^{*}$. It is here that the definition (6) plays a critical role.

Thus, the eigenvectors are

$$
R_{n}{ }^{*} l(y)=n^{*-2}\left[\beta^{3} \Gamma(n-l-I) / 2 \Gamma\left(n^{*}+l^{*}+1\right)\right]^{1 / 2}\left(y / n^{*}\right)^{l^{*}} e^{-y / 2 n^{*}} L_{n-l-I-1}^{(2 l *}\left(y / n^{*}\right)
$$

with eigenenergies given by Eq. (1). The solution still involves the associated Laguerre polynomials because $n^{*}-l^{*}-1$ is equal to $n-l-I-1$, an integer. The eigenvectors (11) are therefore normalizable, and in our convention they obey the orthonormality condition

$$
\int_{0}^{\infty} d r r^{2} R_{n * l *} R_{n * l *}=\delta_{n * n^{* \prime}} .
$$

The usual spectroscopic notation designates energy levels as though they are hydrogenic, so that the wave functions are expected to have $n-l-1$ nodes. This means that the number of nodes in our wave functions can be unusual, since it is given by the order of the associated Laguerre polynomial, $n-l-I-1$. Thus, for example, if $I=0$ or 1 the physical ground state of the $l=0$ lithium Rydberg series, designated as the $2 s$ level, would have one or zero nodes, respectively, even though $n^{*}=1.588$. The node counting is important for an interpretation of the phenomenologically determined integer $I$, as is discussed below.

The solution (11) may be used to calculate expectation values. Due to the factor $n^{*}$ in the expression $\rho=y / n^{*}$, it is easiest to perform the integration using the expression (6) for the associated Laguerre polynomials in terms of products of $\Gamma$ functions. For a transition from the level $\left(n_{i}, l_{i}\right)$ to the level $\left(n_{f}, l_{f}\right)$, the expectation value of $y^{d}=(\beta r)^{d}$ is

$$
\begin{aligned}
\left\langle n_{f}^{*}, l_{f}^{*}\left|y^{d}\right| n_{i}^{*}, l_{i}^{*}\right\rangle= & \frac{1}{2}\left(n_{i}^{*} n_{f}^{*}\right)^{-2}\left(n_{i}^{*}\right)^{-l_{i}^{*}}\left(n_{f}^{*}\right)^{-l_{f}^{*}} F_{n}^{l_{i}^{*}+l_{f}^{*}+d+3}\left[\Gamma\left(n_{i}^{*}-l_{i}^{*}\right) \Gamma\left(n_{f}^{*}-l_{f}^{*}\right) \Gamma\left(n_{i}^{*}+l_{i}^{*}+1\right) \Gamma\left(n_{f}^{*}+l_{f}^{*}+1\right)\right]^{1 / 2} \\
\times \sum_{p=0}^{n_{i}^{*}-l_{i}^{*}-1} \sum_{q=0}^{n_{f}^{*}-l_{f}^{*}-1}\left(-F_{n} / n_{i}^{*}\right)^{p}\left(-F_{n} / n_{f}^{*}\right)^{q} & \\
\times \Gamma\left(l_{i}^{*}+l_{f}^{*}+d+p+q+3\right) /\left[\Gamma(p+1) \Gamma(q+1) \Gamma\left(n_{i}^{*}-l_{i}^{*}-p\right) \Gamma\left(n_{f}^{*}-l_{f}^{*}-q\right)\right. & \vdots \\
\times & \left.\times \Gamma\left(2 l_{i}^{*}+p+2\right) \Gamma\left(2 l_{f}^{*}+q+2\right)\right],
\end{aligned}
$$

where $F_{n}=2 n_{i}^{*} n_{f}^{*} /\left(n_{i}^{*}+n_{f}^{*}\right)$. As required, Eq. (13) satisfies the orthornormality condition (12).

Observe that Eq. (13) has $\left(n_{i}^{*}-l_{i}^{*}\right) \times\left(n_{f}^{*}-l_{f}^{*}\right)$ closedform terms, each of which is a product of $\Gamma$ functions. Therefore, all of our subsequent calculations can in principle be done by hand, on the back of an (admittedly large) envelope.

We may test the eigenvectors (11) by computing transition probabilities for selected atoms. The transition probability $T_{f i}$ for the transition of a valence electron from the level $\left(n_{i}, l_{i}\right)$ to the level $\left(n_{f}, l_{f}\right)$ is 4

$$
\begin{aligned}
T_{f i}= & \left(\pi \alpha^{3} v_{R} / 6\right)\left[\left(n_{f}^{*}\right)^{-2}-\left(n_{i}^{*}\right)^{-2}\right]^{3} \\
& \times F_{l}\left|\left\langle n_{f}^{*}, l_{f}^{*}|y| n_{i}^{*}, l_{i}^{*}\right\rangle\right|^{2},
\end{aligned}
$$

where the fine-structure constant $\alpha$ is given by $\alpha=e^{2} / \hbar c$, the Rydberg frequency $v_{R}$ is given by $v_{R}=\mu e^{4} / 4 \pi \hbar^{3}$, and where $F_{l}$ is an $l$-dependent factor given by

$$
F_{l}=\left\{\begin{array}{l}
l_{i} /\left(2 l_{i}+1\right), \quad l_{f}=l_{i}-1 \\
\left(l_{i}+1\right) /\left(2 l_{i}+1\right), \quad l_{f}=l_{i}+1 .
\end{array}\right.
$$

We have used Eq. (14) to compute probabilities of transition between a variety of levels of the elements lithium and sodium. The $s \rightarrow p$ and $p \rightarrow s$ transition probabilities should provide the most stringent test of the model, because they involve the largest quantum defects. For higher values of $l$, the asymptotic quantum defects are zero, so the eigenstates become hydrogenic.

The value of the integer $I(l)$ was varied with $l$ and was thus selected separately for the initial and final states. The allowed range of variation of $I(l)$ is determined by two conditions. First, requiring the kinetic and potential energies to be separately normalizable imposes the constraint $l^{*}+\frac{1}{2}>0$, i.e., $\delta(l)-l-\frac{1}{2}<I(l)$. Second, the Laguerre polynomials $L_{n-l-I-1}^{\left(2 l^{*}+1\right)}\left(y / n^{*}\right)$ defined by Eq. (6) exist only for $I(l) \leqq n_{\min }-l-1$, where $n_{\min }$ is the principal quantum number of the physical ground state. Thus, for example, for the lithium $s$ orbitals $-0.1<I(l) \leqq 1$; therefore, $I(l)$ can only take the values of 0 and 1 .

Table I lists the results for lithium, along with accepted transition probabilities. ${ }^{7}$ The asymptotic quantum defects $^{2}$ used in our calculations were $\delta(0)=0.40$, $\delta(1)=0.05$, and $\delta(2)=0$. The listed probabilities $T_{f i}$ were obtained with $I(l)=0$ for all $l$; this gave the best agreement with accepted values.

In considering Table $I$, we defer until later a discussion of the special cases of the $2 p \rightarrow 2 s, 3 p \rightarrow 2 s$ transitions and the transition probabilities of order $10^{3} \mathrm{~Hz}$. Then, our results always agree with the accepted values to within $10 \%$ and in certain cases to within a fraction of a percent. Now, the accepted values, except for $2 p \rightarrow 2 s$, are considered valid to within about $10 \% .^{7}$ Therefore, our results are surprisingly good.

In particular, due to the agreement of the $p \rightarrow d$ transi- 
TABLE I. A comparison of our transition probabilities with currently accepted values (Ref. 5) for the lithium atom. The asymptotic quantum defects are $\delta(0)=0.40, \delta(1)=0.50$, and $\delta(2)=0$.

\begin{tabular}{|c|c|c|}
\hline Transition & $\begin{array}{c}\text { Phenomenological } \\
\text { approximation }\left(10^{8} \mathrm{~Hz}\right)\end{array}$ & $\begin{array}{c}\text { Accepted } \\
\text { value }\left(10^{8} \mathrm{~Hz}\right)\end{array}$ \\
\hline $3 s \rightarrow 2 p$ & 0.337 & 0.349 \\
\hline $4 s \rightarrow 2 p$ & 0.106 & 0.101 \\
\hline $4 s \rightarrow 3 p$ & 0.0744 & 0.0746 \\
\hline $5 s \rightarrow 2 p$ & 0.0486 & 0.0460 \\
\hline $5 s \rightarrow 3 p$ & 0.0285 & 0.0276 \\
\hline $5 s \rightarrow 4 p$ & 0.0224 & 0.0225 \\
\hline $2 p \rightarrow 2 s$ & 0.327 & 0.372 \\
\hline $3 p \rightarrow 2 s$ & 0.0170 & 0.0117 \\
\hline $3 p \rightarrow 3 s$ & 0.0356 & 0.0377 \\
\hline $4 p \rightarrow 2 s$ & 0.0169 & 0.0142 \\
\hline $4 p \rightarrow 3 s$ & $9.07 \times 10^{-5}$ & $3.69 \times 10^{-5}$ \\
\hline $4 p \rightarrow 4 s$ & 0.00752 & 0.00772 \\
\hline $4 p \rightarrow 3 d$ & 0.00533 & 0.00552 \\
\hline $5 p \rightarrow 3 d$ & 0.00224 & 0.00231 \\
\hline $5 p \rightarrow 4 d$ & 0.00279 & 0.00286 \\
\hline $6 p \rightarrow 3 d$ & 0.00116 & \\
\hline $6 p \rightarrow 4 d$ & 0.00136 & 0.00139 \\
\hline $6 p \rightarrow 5 d$ & 0.00139 & 0.00142 \\
\hline $3 d \rightarrow 2 p$ & 0.732 & 0.716 \\
\hline $3 d \rightarrow 3 p$ & $6.04 \times 10^{-5}$ & $3.81 \times 10^{-5}$ \\
\hline $4 d \rightarrow 2 p$ & 0.248 & 0.230 \\
\hline $4 d \rightarrow 3 p$ & 0.0702 & 0.0685 \\
\hline $4 d \rightarrow 4 p$ & $1.89 \times 10^{-5}$ & $1.28 \times 10^{-5}$ \\
\hline $5 d \rightarrow 2 p$ & 0.116 & 0.106 \\
\hline $5 d \rightarrow 3 p$ & 0.0361 & \\
\hline $5 d \rightarrow 4 p$ & 0.0138 & 0.0136 \\
\hline $5 d \rightarrow 5 p$ & $6.83 \times 10^{-6}$ & $4.78 \times 10^{-6}$ \\
\hline
\end{tabular}

tions (within 4\%) and the $d \rightarrow p$ transitions (within $10 \%$, except for probabilities of order $10^{3} \mathrm{~Hz}$ ), we consider our phenomenological values of $1.16 \times 10^{5} \mathrm{~Hz}$ for $6 p \rightarrow 3 d$ and $3.61 \times 10^{6} \mathrm{~Hz}$ for $5 d \rightarrow 3 p$ as predictions to be tested against detailed atomic calculations. Other predictions are also made by our model, but the ones quoted involve lower-lying levels and so provide the best test of its predictive power.

Table II contains a similar listing for sodium. In this case, we used asymptotic quantum defects ${ }^{2} \delta(0)=1.35$, $\delta(1)=0.859$, and $\delta(2)=0.01$. The best match to accepted values was obtained with $I(l)$ values of $I(0)=2, I(1)=1$, and $I(2)=0$.

Here, the agreement with accepted values might even be viewed as better than that of Table I. This is because (except for the $3 p \rightarrow 3 s$ transition) the accepted values are all considered $^{7}$ valid to only about $25 \%$ and (except for the $3 p \rightarrow 3 s$ case) all of our values are within this limit. Therefore, our results for the $5 s \rightarrow 4 p, 6 s \rightarrow 5 p, 5 p \rightarrow 5 s$, and $5 d \rightarrow 5 p$ transition probabilities can again be considered as phenomenological predictions.

Returning now to the special cases, note that our caluclations have not allowed for the experimentally observed variation of $\delta(l)$ with $n$, which is largest for small values of $n$. Therefore, low-lying levels should be modeled less accurately than those with larger values of $n$. However, phenomenological quantum defects $\delta_{n}(l)$ that vary slightly with $n$ for a given $l$ may be used just as easily to describe the wave functions, although the number of input parameters becomes large and the exact orthonormality relation is gone. We have therefore calculated transition probabilities using wave functions involving the phenomenological quantum defects $\delta_{n}(l){ }^{2}$ We find that significant improvement in the agreement occurs for the transition probabilities that are small, i.e., of order $10^{3}$ $\mathbf{H z}$, in Table I. The cancellations in the overlap integrals are evidently unusually sensitive to the wave functions involved. Similarly, there are also improvements in the $2 p \rightarrow 2 s$ and $3 p \rightarrow 2 s$ values of Table $I$ and the $3 p \rightarrow 3 s$ value of Table II. The relatively large deviation of the "exact" quantum defects of the lowest levels from the phenomenological quantum defects can be important.

The node structure implied by the values of $I(l)$ for sodium is especially interesting, indicating as it does a distinct departure from the standard hydrogenic picture. Since the number of nodes is $n-l-I-1$, the $s$ orbitals have wave functions with two fewer nodes than expected, while the $p$ orbitals have wave functions with one fewer node.

This situation is reminiscent of supersymmetry. ${ }^{8}$ Our results cannot be used as evidence either for or against supersymmetry, however, because the effective potential 
TABLE II. A comparison of our transition probabilities with currently accepted values (Ref. 5) for the sodium atom. The asymptotic quantum defects are $\delta(0)=1.35, \delta(1)=0.859$, and $\delta(2)=0.01$.

\begin{tabular}{lcc}
\hline \hline Transition & $\begin{array}{c}\text { Phenomenological } \\
\text { approximation }\left(10^{8} \mathrm{~Hz}\right)\end{array}$ & $\begin{array}{c}\text { Accepted } \\
\text { value }\left(10^{8} \mathrm{~Hz}\right)\end{array}$ \\
\hline $4 s \rightarrow 3 p$ & 0.247 & 0.251 \\
$5 s \rightarrow 3 p$ & 0.059 & 0.072 \\
$5 s \rightarrow 4 p$ & 0.0552 & \\
$6 s \rightarrow 3 p$ & 0.0253 & 0.0330 \\
$6 s \rightarrow 4 p$ & 0.0159 & 0.0173 \\
$6 s \rightarrow 5 p$ & 0.0167 & \\
$3 p \rightarrow 3 s$ & & 0.629 \\
$4 p \rightarrow 3 s$ & 0.453 & 0.0292 \\
$4 p \rightarrow 4 s$ & 0.0261 & 0.062 \\
$5 p \rightarrow 3 s$ & 0.057 & 0.0060 \\
$5 p \rightarrow 4 s$ & 0.0060 & 0.0074 \\
$5 p \rightarrow 5 s$ & 0.0073 & \\
$4 p \rightarrow 3 d$ & 0.0129 & 0.00157 \\
$3 d \rightarrow 3 p$ & & \\
$4 d \rightarrow 3 p$ & 0.00189 & 0.495 \\
$4 d \rightarrow 4 p$ & & 0.131 \\
$5 d \rightarrow 3 p$ & 0.421 & 0.067 \\
$5 d \rightarrow 4 p$ & 0.106 & 0.050 \\
$5 d \rightarrow 5 p$ & 0.062 & 0.0260 \\
\hline \hline
\end{tabular}

$V_{\text {eff }}$ in (8) masks the original potential. One cannot tell whether the potential should be $l(l+1) / y^{2}+V_{\text {eff }}$ or $(l+I)(l+I+1) / y^{2}+V_{\text {eff. }}^{\prime}$ The relative sizes of the two constituent pieces of the potential do not provide any clue, as they vary considerably with $l$. One way to tell the origin of the $I(l)$ factors would be to obtain the form of $V_{\text {eff }}$ from a few assumptions about the nature of the electronelectron interactions that $V_{\text {eff }}$ partially models.

In conclusion, the agreement obtained suggests that the analytical eigenvectors (11) may provide good trial wave functions for detailed atomic calculations. ${ }^{9}$ The model may also serve as a phenomenological description that predicts certain previously unknown transition probabilities.

We wish to thank Hans A. Bethe, L. A. Collins, Jeffrey Fuhr, H. Hofmann, Yong-Ki Kim, David R. Mariani, D. Rodney Truax, and, especially, Robert D. Cowan and A. L. Merts for helpful comments.
*Present address: Department of Physics, Indiana University, Bloomington, IN 47405. Address from January to December 1986: Theory Division, CERN, 1211 Genève 23, Switzerland.

${ }^{\dagger}$ Present address (until July 1986): The Niels Bohr Institute, Blegdamsvej 17, 2100 Københaven $\emptyset$, Denmark.

1J. R. Rydberg, Kongl. Sven. vetensk.-akad. hand. 23, no. 11 (1890); Philos. Mag. 29, 331 (1890).

${ }^{2}$ H. G. Kuhn, Atomic Spectra, 2nd ed. (Academic, New York, 1969).

${ }^{3}$ R. D. Cowan, The Theory of Atomic Structure and Spectra (University of California, Berkeley, 1981), pp. 193-199, 218-219, and 224-235. Detailed articles are C. H. Greene, U. Fano, and G. Strinati, Phys. Rev. A 19, 1485 (1979); C. H. Greene, A. R. P. Rau, and U. Fano, ibid. 26, 2441 (1982); M. J. Seaton, Rep. Prog. Phys. 46, 167 (1983).

${ }^{4}$ H. A. Bethe and E. Salpeter, Quantum Mechanics of One- and Two-Electron Atoms (Academic, New York, 1957).

${ }^{5}$ Equation (8) may be viewed as an extension of the BatesDamgaard method, which uses integer $l$ but noninteger $n *$. See D. R. Bates and A. Damgaard, Philos. Trans. R. Soc. London 242, 101 (1949).

${ }^{6}$ That noninteger values of $n^{*}$ and $l^{*}$ can yield analytical solu- tions to the radial equation has been known at least since the work of L. Infeld and T. E. Hull, Rev. Mod. Phys. 23, 21 (1951). Aspects of this problem, including the extension to complex numbers, have been considered in O. Bely, Proc. Phys. Soc. 88, 833 (1966); and J. Dubau, J. Phys. B 11, 4095 (1978). However, our use of analytical wave functions to calculate transition probabilities for bound states seems to be new.

${ }^{7}$ W. L. Wiese, M. W. Smith, and B. M. Glennon, Atomic Transition Probabilities, Vol. I, Natl. Bur. Stand. (U.S.) Natl. Stand. Ref. Data Ser. No. 4 (U.S. GPO, Washington, D.C., 1966); Atomic Transition Probabilities, Vol. II, Natl. Bur. Stand. (U.S.) Natl. Stand. Ref. Data Ser. No. 22 (U.S. GPO, Washington, D.C., 1969).

${ }^{8}$ V. A. Kostelecký and M. M. Nieto, Phys. Rev. Lett. 53, 2285 (1984); Phys. Rev. A 32, 1293 (1985).

${ }^{9}$ Oscillator strengths similar to ours have been obtained by R. E. H. Clark and A. L. Merts (unpublished), for many atomic ions. They treat $\delta_{n}(l)$ as an empirical parameter for the asymptotic wave functions and integrate in to the origin from infinity. 